\title{
The Impact of Increased Hydrocodone Regulation on Opioid Prescribing in an Urban Safety-Net Health Care System
}

\author{
Thomas F. Northrup, PhD, Kelley Carroll, MD, Robert Suchting, PhD, \\ Yolanda R. Villarreal, PhD, Mohammad Zare, MD, and Angela L. Stotts, PhD
}

Background: Hydrocodone-combination analgesics were changed from Schedule III to Schedule II to discourage the prescribing of these analgesics. Our primary aim was to explore the effect of hydrocodone rescheduling on opioid prescribing within an urban safety-net health care system.

Methods and Design: Data were extracted from electronic records of ambulatory patients $(\mathrm{N}=$ 82,432 patients) prescribed hydrocodone-combination, codeine-combination, or tramadol opioid analgesics $(N=200,675$ prescriptions $)$ between 0ctober 6, 2013 and October 6, 2015. Data analyses modeled predicted probabilities of hydrocodone-combination prescriptions (HCPs). Chronic opioid therapy (COT) for chronic pain (ie, $\geq 3$ opioid prescriptions/12 months) and morphine milligram equivalency (MME) levels were also examined.

Results: The probability of providers writing HCPs decreased significantly from pre- to postrescheduling $(0.525$ vs 0.158 , respectively, $P<.0001)$. However, this coincided with large probability increases in codeine-combination $(0.064$ vs 0.269$)$ and tramadol prescriptions $(0.412$ vs 0.573$)$. The probability of HCPs varied across physician specialty $(P<.0001)$, patient diagnoses $(P<.0001)$, COT versus non-COT patients $(P<.0001)$, and patient characteristics (sex, race/ethnicity, and age; $P<$ $.05)$. COT patients received significantly more opioid prescriptions in the postrescheduling period $(M=$ 4.81 vs $M=4.27 ; P<.0001)$. Patients on $<20 \mathrm{MME} /$ day increased slightly from pre- to postrescheduling $(P<.0001)$.

Discussion: Significant declines in HCPs occurred after rescheduling; however, one third of patients prescribed opioids remained on doses $\geq 20 \mathrm{MME} /$ day. Codeine- and tramadol-prescription probabilities increased significantly and providers may have an increased perception of safety about these medications. Physicians and health care systems must reduce their overreliance on opioids in treating pain, especially chronic pain, as all opioids incur some level of risk.(J Am Board Fam Med 2019;32: 362-374.)

Keywords: Codeine-Combination, Hydrocodone, Hydrocodone-Combination, MME, Opioid Prescription, Rescheduling, Tramadol

Opioid-prescribing rates are significantly greater compared with 1999 and earlier, ${ }^{1,2}$ despite recent declines $^{3}$ (eg, 81.2 prescriptions per 100 persons in 2010 dropped to 70.6 in $2015^{2}$ ). Unfortunately,

This article was externally peer reviewed.

Submitted 3 December 2018; revised 17 January 2019; accepted 23 January 2019.

From the Department of Family and Community Medicine, University of Texas Health Science Center at Houston (UTHealth) McGovern Medical School, Houston, TX (TFN, KC, YRV, MZ, ALS); Ambulatory Care Services, Grady Health System, Atlanta, GA (KC); Department of Psychiatry and Behavioral Sciences, UTHealth McGovern Medical School, Houston, TX (RS, ALS). effective multi-modal treatment for chronic pain is often difficult to access for many patients. ${ }^{4}$ Thus, prescribers have limited alternatives to prescribing opioid analgesics to patients who report significant unresolved pain.

Funding: Writing time for TN and AS was supported in part by the National Institute on Drug Abuse (NIDA; R34DA041465; PI: A.L. Stotts) and the NIDA Center for Clinical Trials Network (UG1 DA020024; PI: M. Trivedi) through the U.S. National Institutes of Health and Department of Health and Human Services.

Conflict of interest: none declared. 
It is well established that higher opioid doses and prescribing are associated with increases in opioidrelated overdoses and deaths $s^{5-9}$ and incidence of opioid use disorder (OUD). ${ }^{10}$ For example, hydrocodone-combination analgesics accounted for 97,183 abuse-related emergency department visits in 2011 (96\% increase since 2004). ${ }^{11}$ Drug overdose deaths tripled from 1999 to 2014, with $61 \%$ of those deaths involving an opioid, ${ }^{9}$ most of which were unintentional. ${ }^{12}$ In response to this crisis, hydrocodone-combination analgesics were rescheduled by the US Drug Enforcement Agency (DEA) from Schedule III to Schedule II of the Controlled Substances Act (effective October 6, 2014). ${ }^{13}$ As drug-Schedule levels increase (from I to V), drugabuse and -dependence potential declines. The US DEA defines Schedule II drugs/substances/chemicals as, "drugs with a high potential for abuse, with use potentially leading to severe psychological or physical dependence." 14 Rescheduling hydrocodone-combination analgesics to Schedule II intentionally made prescribing these analgesics more difficult (eg, refills are not allowed on Schedule II) and encouraged more physician oversight. ${ }^{14}$

In 2016, the US Centers for Disease Control and Prevention (CDC) released guidelines for managing patients with chronic pain. ${ }^{15}$ Specifically, CDC guidelines recommend calculating morphine milligram equivalency (MME) per day (MME/day) and MME per prescription (MME/prescription), for potency comparisons across opioids. ${ }^{5,16}$ Ideally patients are prescribed $<20 \mathrm{MME} /$ day, as health risks increase between 20 and $50 \mathrm{MME} /$ day, and escalate further at $\geq 50 \mathrm{MME} /$ day. ${ }^{5,6,8,10}$

Overall, the number of physician-written hydrocodone-combination prescriptions (HCPs) and dispensed tablets decreased by $22.0 \%$ and $16.0 \%$, respectively, following rescheduling. ${ }^{3}$ Our initial work also identified reductions in HCPs in the 6 months after rescheduling. ${ }^{17}$ The analysis of IMS Health National Prescription Audit data reported a slight uptick in dispensed nonhydrocodone combination opioid analgesic prescriptions (eg, codeinecombination products; $4.9 \%$ ) and tablets $(1.2 \%){ }^{3}$

Corresponding author: Thomas F. Northrup, PhD, Department of Family and Community Medicine, The University of Texas Health Science Center at Houston (UTHealth), McGovern Medical School, 6431 Fannin, JJL 324, Houston, TX, 77030 (E-mail: Thomas.F.Northrup@uth.tmc.edu).
Codeine-combination drugs remain Schedule III analgesics. ${ }^{14}$

Interestingly, past research on rescheduling changes, such as the IMS analysis, has not included tramadol. Tramadol has been associated with lower to similar abuse potential compared with medications such as buprenorphine, but higher abuse potential compared with placebo ${ }^{18}$; this has led to higher regulatory control in non-US countries. ${ }^{19}$ In the US, tramadol is currently a Schedule IV analgesic ${ }^{14}$ and accounting for tramadol prescriptions is essential to fully understanding how HCP regulation affected physician-opioid-prescribing behavior.

Further, several areas related to physician-opioid-prescribing behavior remain underexplored after rescheduling. First, substitution of one opioid analgesic for another across the rescheduling period (eg, from hydrocodone- to codeine-combination or tramadol-based analgesics) has not been explored at the patient level. Studying this may elucidate how responsive physicians were to rescheduling, as well as provide data on what proportion of patients were affected by prescription substitution. As suggested by past research, providers may be less likely to change HCPs for certain primary diagnoses; and, women, members of racial/ ethnic minorities, and the elderly have been shown to receive differential pain treatment. ${ }^{20}$ Therefore, we examined pre- to postrescheduling changes in HCP probabilities by patient diagnoses, patient characteristics (sex, race, age), and medical specialties, to further understanding of these relationships. Exploration of these complex physician and patient characteristics may improve pain management and indicate which patients may be at higher risk for opioid-related risks.

Our primary aim was to identify HCP-probability changes after rescheduling, within ambulatory settings of a large, urban, safety-net system, and explore patient/provider characteristics associated with changes. An important secondary aim included describing providers' changes in codeinecombination and tramadol-analgesic prescribing and we explored opioid-related risks based on within-patient MME comparisons across the rescheduling periods. Based on our initial work with shorter timeframes, ${ }^{17}$ we hypothesized that providers would issue fewer HCPs, while issuing compensatory increases in codeine-combination and tramadol prescriptions. 


\section{Methods}

The UTHealth McGovern Medical School Committee for the Protection of Human Subjects, and the health care system both approved this retrospective electronic-medical-record (EMR) review (HSC-MS-15-0517).

\section{Health care Setting}

The large, urban safety-net health care system includes 18 outpatient community health clinics, 5 school-based clinics, 5 specialty clinics, 5 urgentcare (same-day) clinics, 3 small clinics for homeless individuals, and 3 county hospitals. Noteworthy, this health system improved access to care (eg, more primary care providers were hired and several urgent-care centers were newly opened) in 2014 but overall unduplicated patients did not increase significantly. Data were restricted to ambulatory settings only, which served $>227,000$ unduplicated patients in 2016. Across this health care system, patients were predominantly female $(62 \%)$, from an ethnic/racial minority (eg, 53.3\% Hispanic; $19.6 \%$ African-American), and self-pay (68.4\%) or on Medicaid (13.2\%) or Medicare (7.2\%).

\section{Participants and Measures}

All patients with $\geq 1$ prescription(s) for hydrocodone-combination, codeine-combination, or tramadol opioid analgesics were included $(\mathrm{N}=$ 200,695 prescriptions; $\mathrm{N}=82,432$ patients). EMR data were abstracted from 12 months before and 12 months after hydrocodone rescheduling from Schedule III to Schedule $\mathrm{II}^{13}$ (range: October 6, 2013 to October 6, 2015).

Only hydrocodone-combination, codeine-combination, and tramadol formulations were studied as they comprised $>99 \%$ of ambulatory opioid prescriptions in this health system. A subset of patients (see Table 1) filled their prescriptions at a health system-affiliated pharmacy $(n=95,126$ prescriptions filled [ $47.3 \%$ of total prescriptions]; $\mathrm{n}=$ 37,328 patients $[45.3 \%$ of sample]), permitting MME-related analyses on this subset of patients. MME-conversion factors were multiplied by the milligram dose of hydrocodone, codeine, and tramadol per tablet $(1,0,0.15,0.10$, respectively) to obtain MME/tablet, which was multiplied by the total number of tablets/prescription to obtain the total MME a patient has access to (MME/prescription).
Opioid-analgesic changes (ie, substitution) from pre- to postrescheduling were explored in patients with $\geq 1$ opioid prescriptions in each rescheduling period ( $\mathrm{n}=12,481$ patients; $15.1 \%$ of sample). Within patients, we compared the last prerescheduling opioid prescription to the first postrescheduling prescription.

Patients on chronic opioid therapy (COT) for chronic pain were defined as patients with $\geq 3$ opioid prescriptions within a 1 -year period $(n=17,316$; $21.0 \%$ of sample), similar to previous work (eg, pain lasting $>3$ months beyond normal tissue healing ${ }^{21}$ ).

\section{Measures}

Abstracted EMR data included patient characteristics (sex, age, race/ethnicity), prescribing physician specialty, prescription order dates, opioid formulation and tablet-level dose prescribed, dispensed data (date and pill count [if filled by an in-network pharmacy]), and primary ICD-9 diagnosis (see Table 1).

\section{Statistical Analyses}

Generalized estimating equations (GEE) investigated the probability of patients being prescribed a HCP versus other opioid prescription (codeinecombination/tramadol) as a function of rescheduling period (pre/post) in the full sample (Model 1). Medical specialty, diagnosis, sex, race/ethnicity, and age were separately tested as moderators of the relationship between rescheduling period and HCP in Models 2 through 6, respectively. Model 7 examined effects of being on COT on being prescribed a HCP. Generalized estimating equations modeling was utilized to handle nonnormal outcomes (eg, dichotomous binomial outcomes) and account for correlated observations (eg, multiple patient prescriptions) to provide population-averaged estimates. ${ }^{22}$ Descriptive statistics and $\chi^{2}$ tests were utilized to characterize opioid-prescription substitution and patient MME levels.

\section{Results}

Inclusion criteria ( $\geq 1$ hydrocodone-combination, codeine-combination, or tramadol prescription) were met by 82,432 unique patients. Table 1 presents patient-level and prescription-level data for the total sample, by pre- and postrescheduling periods, and separately for patients on and not on COT. 


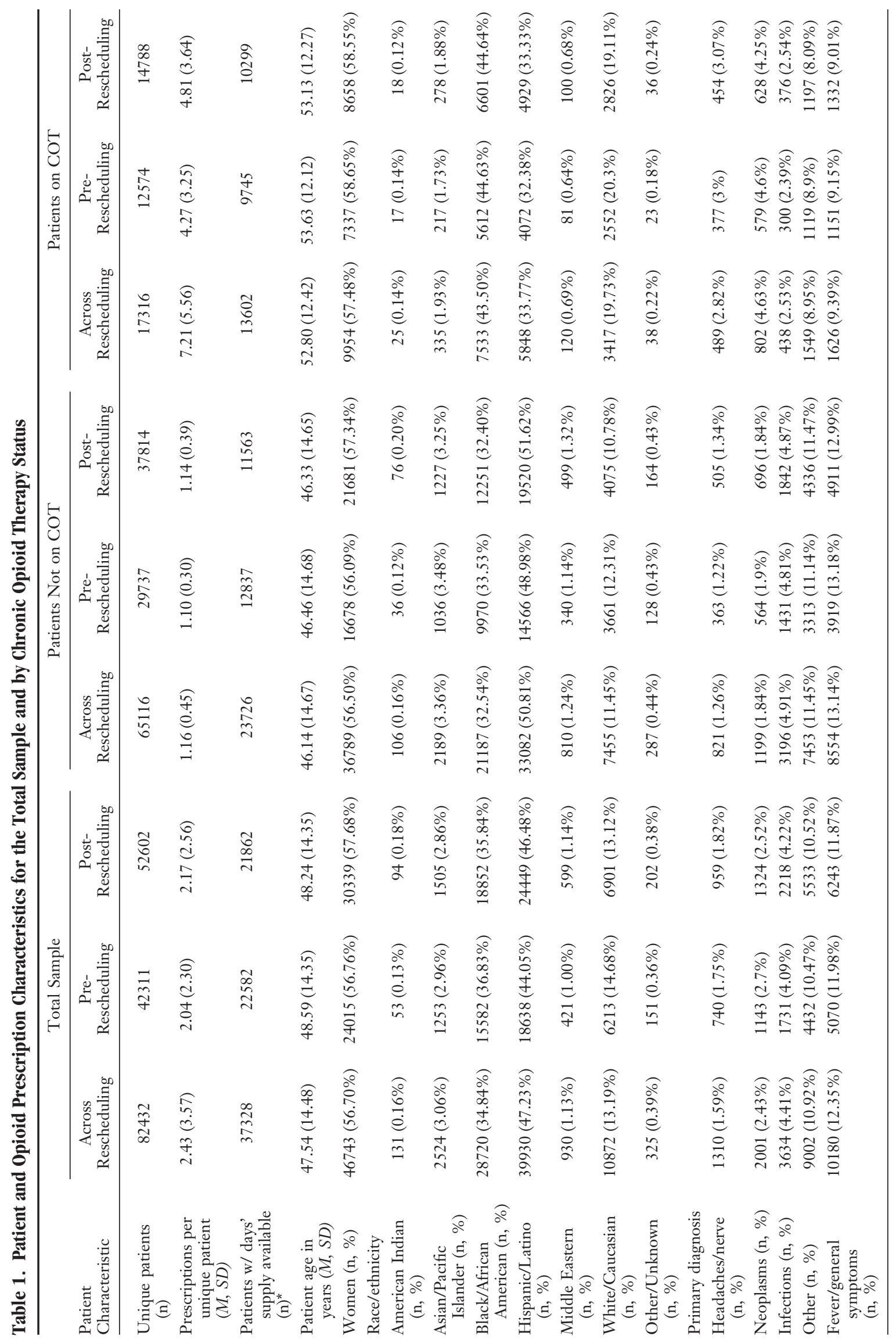




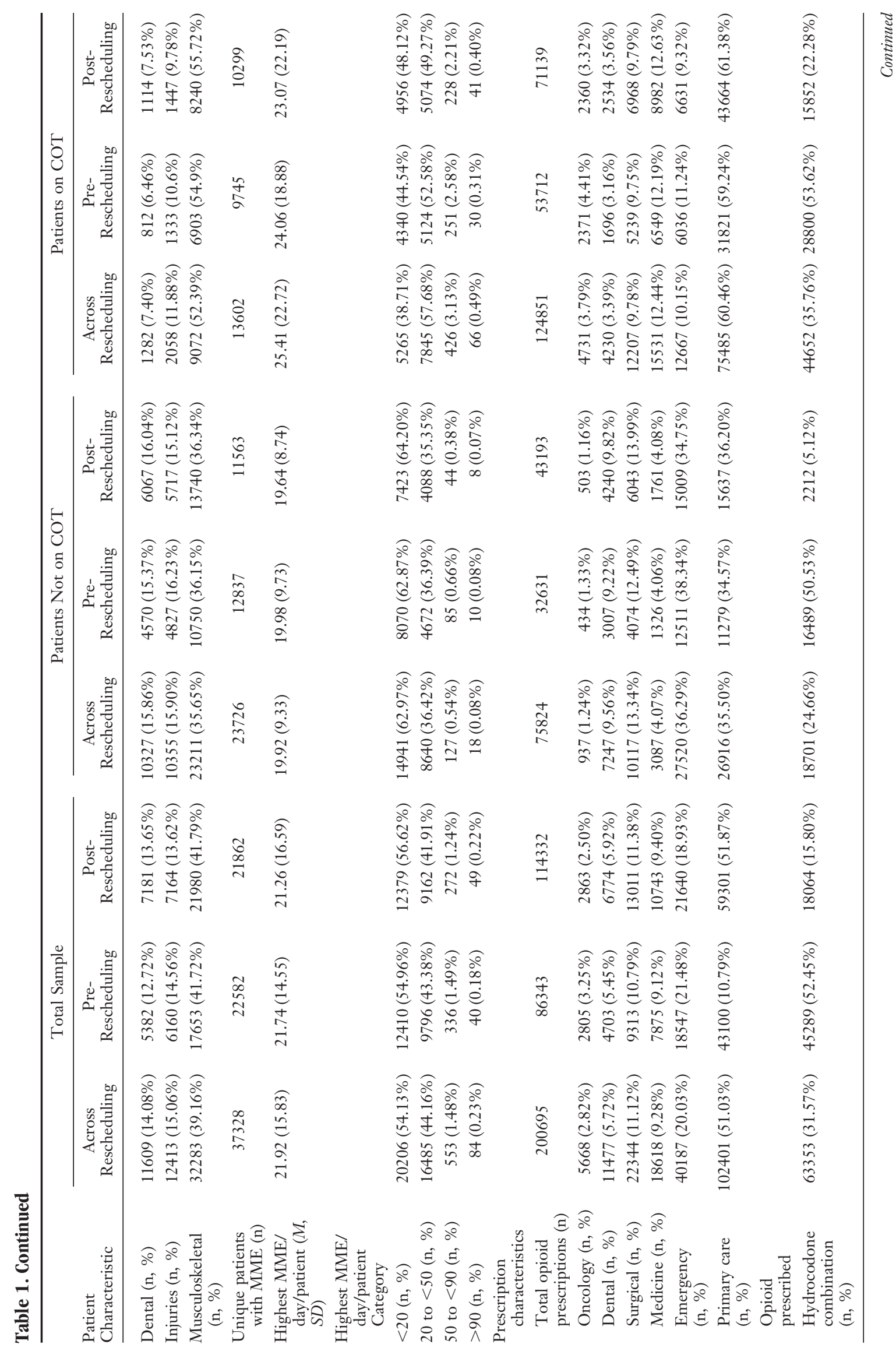




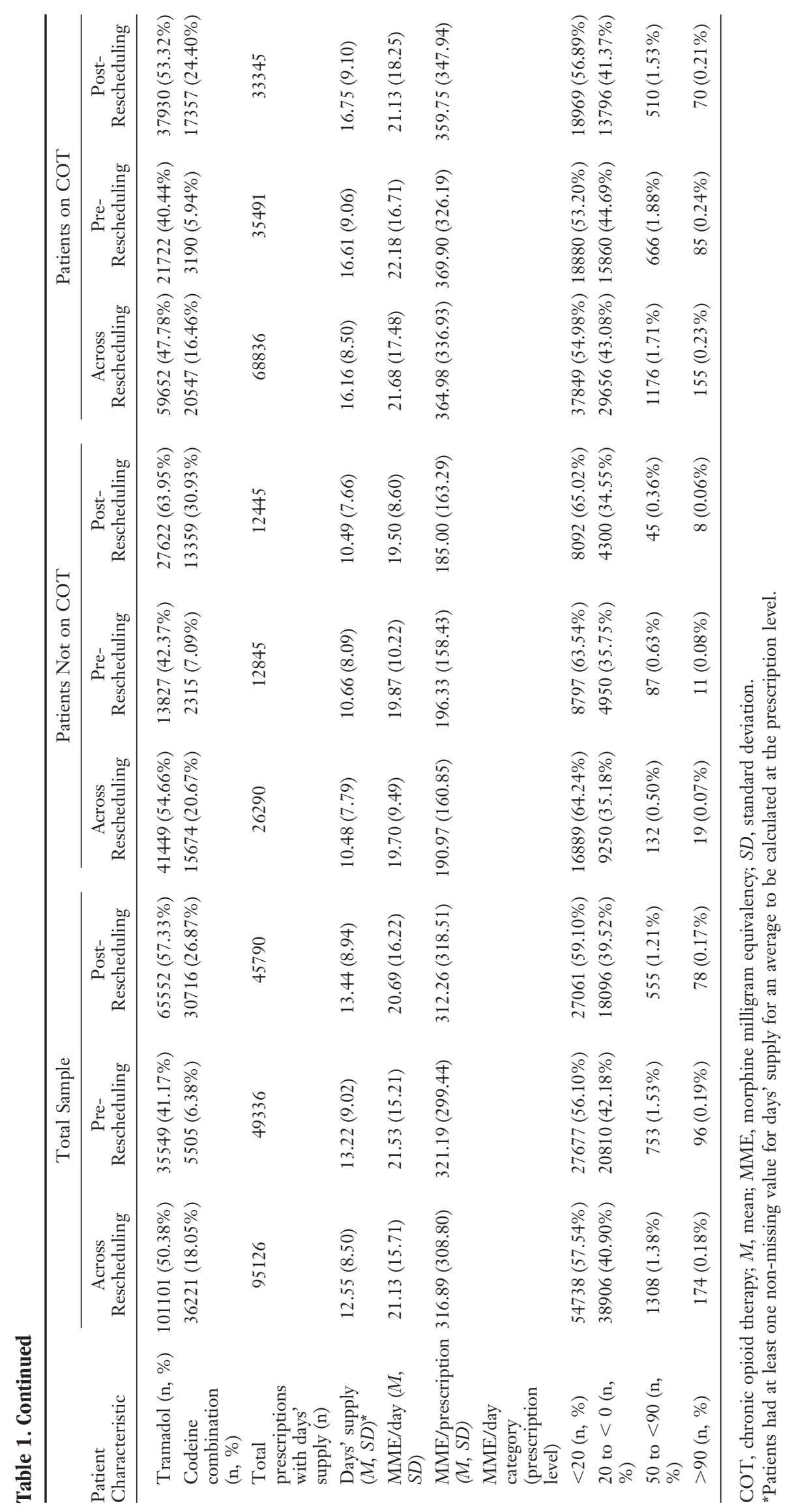


Figure 1. Probability of hydrocodone-combination prescriptions by rescheduling period (A) and by rescheduling period within medical specialty (B), patient diagnoses (C), patient sex (D), patient race/ethnicity (E), and chronic opioid therapy (COT) status (F). Note. For panel E, patients identified as "American Indian, Asian/Pacific Islander, and Middle Eastern" were combined with patients identified as "Other/Unknown" in the electronic-medical-record (EMR) to form "Other," due to relatively low overall frequencies for these race/ethnicities. PCP, primary care physician.
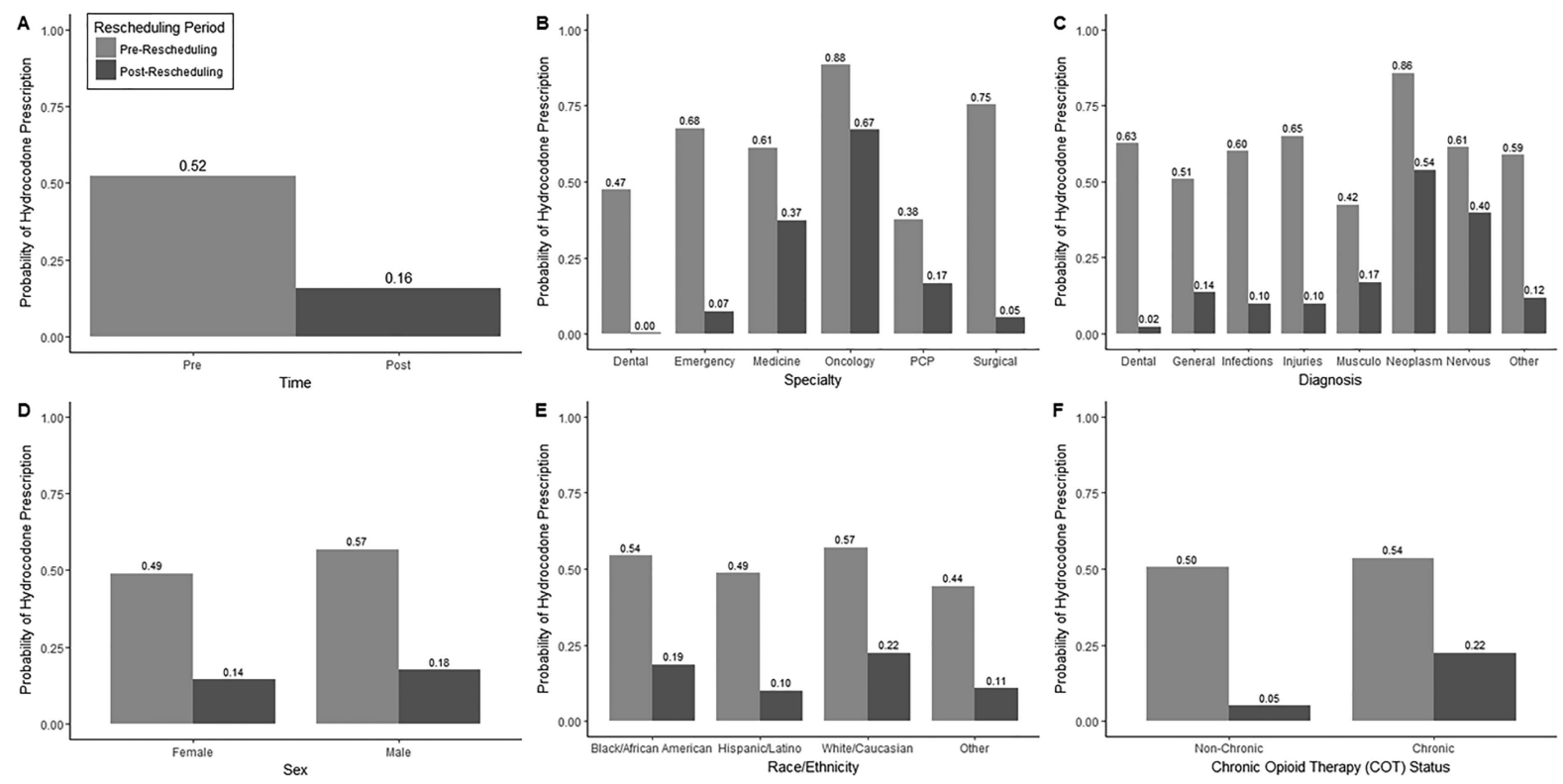

\section{Probability of HCPs by Rescheduling Period}

The predicted probability of being prescribed a HCP (relative to codeine-combination/tramadol prescriptions) decreased significantly from prerescheduling $(0.525)$ to postrescheduling $(0.158 ; P<$ $.0001)$, across all patients (Figure 1A). Tramadol prescriptions nearly doubled and codeine-combination prescriptions increased over 5-fold after rescheduling. Despite a HCP decline, the mean number of opioid prescriptions (ie, any hydrocodone- or codeine-combination or tramadol analgesic) ordered per patient increased from pre- to postrescheduling $(P<.0001$; see Table 1$)$ and $24.3 \%$ more patients were prescribed an opioid in the postrescheduling period, resulting in $32.4 \%$ more opioid prescriptions written in the postrescheduling period. Noteworthy, the mean days' supply per prescription did not change from pre- to postrescheduling (see Table 1).

\section{HCP by Medical Specialty}

Every medical specialty/subspecialty had a significant absolute reduction in the probability of HCPs across rescheduling periods $(P<.0001$ for every specialty) and differential HCP decreases by specialty are shown in Figure 1B $(P<.0001)$. Note- worthy, primary care accounted for $51.0 \%$ of all opioid prescriptions across both periods (see Table 1) and had the lowest prerescheduling HCP probability and 1 of the 3 highest postrescheduling HCP probabilities.

\section{HCP by Diagnoses}

Each diagnostic cluster had a significant absolute reduction in the probability of HCPs across rescheduling $(P<.0001$ all diagnoses $)$, and significant variability in HCP reductions were demonstrated by diagnosis $(P<.05$; Figure $1 \mathrm{C})$. Musculoskeletal diagnoses accounted for $39.2 \%$ of patient diagnoses across both periods (Table 1) and had the lowest prerescheduling HCP probability (Figure 1C). Aside from neoplasms, headaches/ nerve pain had the highest postrescheduling hydrocodone-combination probability and dental-related diagnoses had the lowest postrescheduling HCP probability.

\section{HCP by Sex}

Both men and women had a significant absolute reduction in the probability of HCPs across rescheduling $(P<.0001)$. Women accounted for $56.7 \%$ of patients (Table 1 ) and $57.3 \%$ of all opioid 
Figure 2. Probability of hydrocodone-combination prescription by age in years (centered) for each rescheduling period.

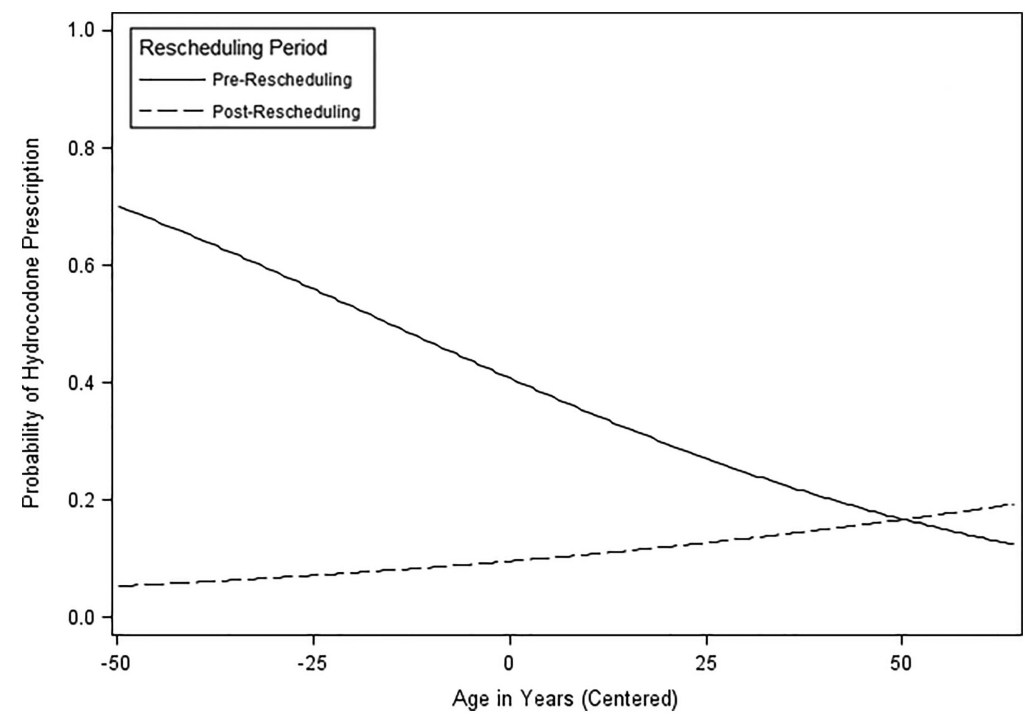

prescriptions across both periods, but had a lower prerescheduling HCP probability (relative to codeine and tramadol analgesics) than men $(P<$ .0001 ; see Figure 1D). Post rescheduling, the magnitude of sex-based HCP differences decreased, with men still having a slightly higher HCP probability than women $(P<.0001)$.

\section{HCP by Race/Ethnicity}

Each race/ethnicity (see Table 1) had a significant absolute reduction in the probability of HCPs across rescheduling $(P<.0001$ for all races/ethnicities) and absolute decreases in HCP probabilities were similar across race/ethnicities but still reached statistical significance (see Figure 1E; $P<.05$ ). Prerescheduling HCP probabilities were greatest for White, non-Hispanic patients, followed by Black/African-American patients, Hispanic/Latino patients, and patients from Asian/Pacific Islander or other races/ethnicities (Figure 1E). Noteworthy are the significant postrescheduling probability differences, with White/white patients having a higher HCP probability, compared with Black/African-American patients $(P<.001)$, Hispanic/Latino patients $(P<.0001)$, and patients of other races/ethnicities $(P<.0001)$. Further, Black/African-American patients were more likely to be prescribed HCPs compared with Hispanic/Latino patients $(P<.0001)$ and patients of other races/ ethnicities $(P<.0001)$.

\section{HCP by Age}

The model testing patient age, included 2 covariates known to correlate with age (ie, neoplasm diagnosis [yes/no] and COT status [yes/no]). Both covariates were statistically significant (each $P<$ .0001). In the prerescheduling period, as age increased, the probability of being prescribed a HCP decreased (see Figure 2). Age demonstrated a significant interaction with rescheduling $(P<.0001)$, and in the postrescheduling period as age increased the HCP probability increased.

\section{Patients on COT for Chronic Pain ( $\geq 3$ Opioid Prescriptions in 12 Months)}

Both COT and non-COT patients had a significant HCP probability reduction across rescheduling (Model 7; $P<.0001$ ) but differential reductions were observed $(P<.0001)$. Before rescheduling, COT patients had significantly fewer total opioid prescriptions compared with the postrescheduling period $(P<.0001$; Table 1$)$ and had similar HCP probabilities to patients not on COT (see Figure 1F). Non-COT patients showed greater HCP probability reductions (see Figure $1 \mathrm{~F}$ ), resulting in significantly lower probabilities of $\mathrm{HCPs}$, compared with COT patients after rescheduling $(P<$ .0001). Noteworthy, the mean days' supply per prescription did not change significantly from preto postrescheduling for COT or non-COT patients (see Table 1). 
Post-hoc analyses revealed key differences across COT and non-COT patients. Across both rescheduling periods, COT patients tended to be women and older than non-COT patients $(t=-54.72$, $P<.0001$; see Table 1). Differences in proportions of races/ethnicities were also found $(P<.0001)$. For example, greater proportions of White/white (19.7\% vs $11.5 \%$, respectively) and African-American/Black patients ( $43.5 \%$ vs $32.5 \%$, respectively) were found among COT patients, compared with non-COT patients $(P<.0001)$.

\section{Within-Patient Opioid Substitutions}

For patients with $\geq 1$ opioid prescription in each rescheduling period ( $\mathrm{n}=12,481$ patients; $15.1 \%$ of total sample), a large minority of patients were prescribed a codeine-combination analgesic or tramadol for their final prerescheduling prescription and received the same prescription (codeinecombination analgesic or tramadol) at their first postrescheduling prescription $(\mathrm{n}=5498$; $44.0 \%$ ). Almost one third of patients switched from a hydrocodone-combination to codeinecombination or tramadol analgesic $(\mathrm{n}=3929$; $31.5 \%)$. Only $13.9 \%(\mathrm{n}=1728)$ remained on a hydrocodone-combination analgesic and only $1.8 \%(n=230)$ switched from a codeine-combination or tramadol analgesic to a hydrocodonecombination analgesic. The remainder $(\mathrm{n}=$ 1096; 8.8\%) switched from a codeine-combination to tramadol or vice versa.

\section{Morphine Milligram Equivalency}

Table 1 provides morphine milligram equivalency (MME) data at the patient and prescription level; results described in this section refer to the highest mean MME/day per patient, except where noted. Before rescheduling, a slight majority were prescribed $<20 \mathrm{MME} /$ day $(54.1 \%)$, with notable differences for non-COT $(62.9 \%)$ and COT patients (44.5\%; $P<.0001)$. Most of the remaining patients were prescribed a dose between 20 and $50 \mathrm{MME} /$ day, and $<1.8 \%$ of all patients were prescribed $>50$ MME/day (with COT patients accounting for a majority of patients on >50 MME/day).

Only a slight decrease was observed in the mean MME/day from pre- to postrescheduling $(P<$ .0001), with non-COT patients having a smaller decrease than COT patients in MME/day (see Table 1). The mean MMEs per prescription followed similar trends.
In the postrescheduling period, marginally more patients were prescribed $<20 \mathrm{MME} /$ day compared with prerescheduling $(56.6 \%$ vs $55.0 \%$, respectively). Differences persisted in the postrescheduling period for the proportion of non-COT patients prescribed $<20 \mathrm{MME}$ /day compared with COT patients $(P<.0001$; see Table 1$)$.

\section{Discussion}

Consistent with previous findings, ${ }^{3}$ US DEA hydrocodone-combination-analgesic scheduling changes led to significant declines in HCPs in this safety-net health care system; however, codeine-combination and tramadol opioid prescriptions rose dramatically, more than offsetting the HCP decreases. While patients' HCP-related risks declined, rescheduling HCPs may have done little to reduce patients overall opioid exposure, as more patients were prescribed opioids in the first 12 months after rescheduling compared with the 12 months before rescheduling. Indeed, the overall number of opioid prescriptions ordered increased by a third and the number of patients prescribed an opioid rose by nearly $25 \%$ after rescheduling. The relatively high proportion of patients prescribed opioids in this system may be partially explained by the specific needs of patients who access care through a safety-net system, who often have lower socioeconomic resources and higher rates of mental illness, chronic pain, and disability than the general population. ${ }^{23,24}$

The increase in number of prescriptions may simply reflect changes in opioid policies (eg, prohibition of HCP refills by telephone), requiring more frequent in-person visits and thus more prescriptions were written. As noted previously, primary care access improved and several urgent care centers were opened during the study time frame in this health system, which may have increased the number of opioid prescriptions to some extent. However, the increase in the number of and proportion of patients being prescribed an opioid after rescheduling cannot fully be explained by these factors.

Codeine-combination (schedule III) and tramadol analgesics (schedule IV) are considered to have lower abuse potential than hydrocodonecombination analgesics (schedule II) ${ }^{14}$ but they are not without risk. In the 6 months after re- 
scheduling (compared with the 6 months before rescheduling), Texas Poison Centers saw hydrocodone-related exposures decline (from 1567 to 1135 cases) but codeine misuse increased significantly (from 189 to 522 cases) and tramadol exposures rose slightly (from 666 to 708 cases). ${ }^{25}$ The increases in codeine- and tramadol-related exposures almost completely wiped away the declines in hydrocodone-related poison calls. Further, relatively modest decreases in MME/day were found in this study, making it uncertain how much (if at all) hydrocodone-related risks may have been mitigated in this health system by rescheduling hydrocodone. This is especially concerning as over $43 \%$ of patients on COT received prescriptions for $\geq 20 \mathrm{MME} /$ day in the postrescheduling period, incurring higher risk for opioid-related harm relative to lower doses. ${ }^{5,8}$ Statewide prescription-monitoring programs are associated with sustained reductions in schedule-II drugs ${ }^{26}$ but monitoring programs are not in use with schedule-III and -IV opioid analgesics. An unintended effect of rescheduling hydrocodone-combination analgesics may be a perception of safety for codeine-combination and tramadol analgesics and perhaps has led to physicians prescribing these opioid formulations more readily.

It is important to address concerns about causing opioid-related harm, ${ }^{27}$ including provider concerns about insufficient training, ${ }^{27,28}$ while minimizing the under treatment of pain and health-related disparities. Our data underscored differential reductions in HCPs across provider specialty and patient diagnoses, complicating our understanding of patients' opioid exposures and risks. Primary care physicians (PCPs) continue to write the majority of opioid prescriptions (including HCPs) and manage the highest proportion of patients on COT. There is great need to help PCPs manage patients on opioids or alternative analgesics with multi-modal interventions for acute and chronic pain. ${ }^{29}$ However, access to comprehensive pain management programs is limited. Health care systems and insurers need to provide access to evidence-based nonopioid pharmacologic and nonpharmacologic alternatives (as reviewed in ${ }^{15}$ and explore other patient safeguards (eg, limit multiple prescribers for individual patients to reduce opioid overdoses ${ }^{30,31}$ ).
Consistent with CDC guidelines, ${ }^{15}$ minimizing MME/day and engaging in evidence-based practices may meaningfully reduce opioid-related harm. Specifically, the CDC Guidelines for Prescribing Opioids for Chronic Pain recommends risk assessment before opioid initiation, use of prescription drug monitoring programs and urine monitoring, avoidance of concurrent benzodiazepines, providing initial therapy without long-acting opioids, prescribing less than 90 MME/day, as well as improving access to OUD treatment. ${ }^{15}$ Policy changes increasing physician oversight of patients' opioid use are likely to have benefits for patient health, while simultaneously increasing health care system and provider burden. For the latter reason, efficacious programs for reducing physicians' and patients' reliance on opioids to manage pain are both attractive and an imperative. As others have noted, it may prove difficult to get providers to change current opioid-prescribing practices, ${ }^{29}$ especially for patients with headaches/nerve and musculoskeletal diagnoses who were more likely to be prescribed HCPs after rescheduling, behind only patients with neoplasms. More research is needed to elucidate the role of opioids in chronic, noncancer pain, and to develop both pharmacological and nonpharmacological alternatives.

Our results added to growing evidence that women, racial/ethnic minorities, and older patients may experience differential pain management. ${ }^{20}$ From a clinical perspective, the postrescheduling differences in HCPs by sex in our dataset were minimal. Differences across race/ ethnicity were more pronounced. Consistent with past research, ${ }^{32-35}$ White, non-Hispanic patients more likely to be prescribed a HCP than Black/African-American patients, and more than twice as likely compared with Hispanic/Latino patients and other races/ethnicities. Ironically, this potential racial bias may have operated in a positive direction, as individuals who died from opioid-analgesic-overdoses in one study were more likely to be White, non-Hispanic, than Black or Hispanic. ${ }^{36}$ White, non-Hispanic and Black/African-American patients comprised an outsized proportion of the patients on COT for chronic pain, despite Hispanic/Latino patients comprising the majority of patients in this health care system. Some of these pain-management differences have been explained by cultural pref- 
erences. ${ }^{37}$ Finally, after accounting for COT status and neoplasm status, the association of patient age with HCP probability differed across rescheduling periods. The probability of a HCP was lower as age increased in the prerescheduling period and greater after rescheduling. This is commensurate with the finding that COT patients tended to be older than non-COT and that COT patients had a greater probability of being prescribed a hydrocodone-combination analgesic. Regardless, these data suggest sex-, race-/ ethnicity-, and age-related health disparities for pain management in need of further study.

This study provided new insights on dramatic increases of codeine and tramadol prescriptions after increased regulation of hydrocodone but was not without limitations. These data came from a single urban, safety-net system, in a non-Medicaid expansion state, which may differ in important ways from others cities/states/regions. We did not have opioid-prescription data for all formulations nor complete pharmacy data for all patients, potentially affecting MME and prescription-level calculations. However, the degree of bias due to opioid exclusions (eg, oxycodone, fentanyl) is likely small, as these opioids are less commonly prescribed in ambulatory settings in this system. In addition, our definition of COT for chronic pain (ie, $\geq 3$ prescriptions) may not fully align with past studies. Conversely, key differences found between nonCOT and COT patients suggested that our definition may provide results similar to other studies' methods for defining patients on COT. ${ }^{21}$ Our analyses did not account for analgesic substitutions from opioid to nonopioid analgesics but we speculate that a number of patients may have switched to nonopioid analgesics or had a nonopioid analgesic prescribed in the postrescheduling period. Future prospective evaluations will improve on these methods and explore whether these significant HCP reductions and opioid-analgesic substitutions have adversely affected patients' pain management (eg, undertreatment ${ }^{17}$ ).

\section{Conclusion}

Our findings show that hydrocodone rescheduling by the US DEA led to significant declines in HCPs, across all specialties, sexes, races/ethnicities, age, diagnoses, and COT status, within this large, safety-net system. The net benefit to patients from the decline in HCPs was potentially offset by significant increases during the 12 months after rescheduling in codeine and tramadol prescriptions, minimal declines in MME/day (per patient), and concerns about race/ethnicity-related pain-management disparities. Further, PCPs continue to bear significant, on-going opioid-prescribing burdens. The risks of developing an OUD, ${ }^{38}$ overdosing, or dying from an opioid overdose for the majority of COT patients continuing to be prescribed $\geq 20 \mathrm{MME} /$ day remain significant public health concerns.

To see this article online, please go to: http://jabfm.org/content/ 32/3/362.full.

\section{References}

1. Rosenblum A, Marsch LA, Joseph H, Portenoy RK. Opioids and the treatment of chronic pain: Controversies, current status, and future directions. Exp Clin Psychopharmacol 2008;16:405-16.

2. Schuchat A, Houry D, Guy GP. New data on opioid use and prescribing in the United States. JAMA 2017;318:425-6.

3. Jones CM, Lurie PG, Throckmorton DC. Effect of US Drug Enforcement Administration's rescheduling of hydrocodone combination analgesic products on opioid analgesic prescribing. JAMA Inter Med 2016;176:399-402.

4. Perry J, VanDenKerkhof EG, Wilson R, Tripp DA. Development of a guided internet-based psycho-education intervention using cognitive behavioral therapy and self-management for individuals with chronic pain. Pain Manag Nurs 2017;18:90101.

5. United States Centers for Disease Control. Calculating total daily dose of opioids for safer dosage. 2018. Available from: https://www.cdc.gov/drugoverdose/pdf/calculating_total_daily_dose-a.pdf. Accessed January 22, 2018.

6. Liang Y, Turner BJ. Assessing risk for drug overdose in a national cohort: Role for both daily and total opioid dose? J Pain 2015;16:318-25.

7. Dunn KM, Saunders KW, Rutter CM, et al. Opioid prescriptions for chronic pain and overdose: A cohort study. Ann Intern Med 2010;152:85-92.

8. Gomes T, Mamdani MM, Dhalla IA, Paterson JM, Juurlink DN. Opioid dose and drug-related mortality in patients with nonmalignant pain. Arch Intern Med 2011;171:686-91.

9. Rudd RA. Increases in drug and opioid-involved overdose deaths-United States, 2010-2015. MMWR Morbid Mortal Wkly Rep 2016;65.

10. Edlund MJ, Martin BC, Russo JE, DeVries A, Braden JB, Sullivan MD. The role of opioid prescription in incident opioid abuse and dependence 
among individuals with chronic noncancer pain: The role of opioid prescription. Clin J Pain 2014; 30:557-64.

11. Center for Behavioral Health Statistics and Quality Substance Abuse and Mental Health Services Administration. The DAWN Report: Highlights of the 2011 Drug Abuse Warning Network (DAWN) findings on drug-related emergency department visits. 2013. Available from: http://www.samhsa.gov/data/ sites/default/files/DAWN127/DAWN127/sr127DAWN-highlights.htm. Accessed January 19, 2018.

12. Miller M, Barber CW, Leatherman S, et al. Prescription opioid duration of action and the risk of unintentional overdose among patients receiving opioid therapy. JAMA Intern Med 2015;175:608-15.

13. US Department of Justice Drug Enforcement Administration, Diversion Control Division. Rules-2014. 2014. Available from: https://www.deadiversion.usdoj.gov/fed_regs/rules/2014/fr0822.htm. Accessed January 18, 2018.

14. United States Drug Enforcement Administration. Drug Scheduling. 2019. Available from: https://www. dea.gov/drug-scheduling. Accessed January 4, 2019.

15. Dowell D, Haegerich TM, Chou R. CDC guideline for prescribing opioids for chronic pain-United States, 2016. JAMA 2016;315:1624-45.

16. United States Department of Health and Human Services, Centers for Disease Control. Opioid oral morphine milligram equivalent (MME) conversion factors. 2016. Available from: https://www.cms.gov/ Medicare/Prescription-Drug-Coverage/Prescription DrugCovContra/Downloads/Opioid-Morphine-EQConversion-Factors-Aug-2017.pdf. Accessed January 22, 2018.

17. Carroll K, Northrup T, Zare M, Stotts A. Dramatic reduction in hydrocodone prescriptions in an urban safety net health care system: Has it improved patient safety or led to increased patient suffering? Pain Med 2016;17:213-6.

18. Das M, Jain R, Dhawan A, Kaur A. Assessment of abuse liability of Tramadol among experienced drug users: Double-blind crossover randomized controlled trial. J Opioid Manag 2016;12:421-30.

19. Jalali S, Thomas D, Shetty P, Cooper Jason C. Higher Regulatory Control of Tramadol to Prevent its Abuse and Dependence. J Drug Policy Analysis 2017. DOI: $10.1515 /$ jdpa-2016-0008.

20. National Research Council. Relieving Pain in America: A Blueprint for Transforming Prevention, Care. Education, and Research. Washington, DC: The National Academies Press. 2011.

21. International Association for the Study of Pain. Classification of chronic pain. Descriptions of chronic pain syndromes and definitions of pain terms. Prepared by the International Association for the Study of Pain, Subcommittee on Taxonomy. Pain Suppl 1986;3:S1-S226.
22. Lee JH, Herzog TA, Meade CD, Webb MS, Brandon TH. The use of GEE for analyzing longitudinal binomial data: A primer using data from a tobacco intervention. Addict Behav 2007;32:187-93.

23. Goodwin L, Gazard B, Aschan L, MacCrimmon S, Hotopf M, Hatch S. Taking an intersectional approach to define latent classes of socioeconomic status, ethnicity and migration status for psychiatric epidemiological research. Epidemiol Psychiatr Sci 2018;27:589-600.

24. Schild C, Reed EA, Hingston T, Dennis CH, Wilson AC. Neighborhood characteristics: Influences on pain and physical function in youth at risk for chronic pain. Children 2016;3:35.

25. Haynes A, Kleinschmidt K, Forrester MB, Young A. Trends in analgesic exposures reported to Texas Poison Centers following increased regulation of hydrocodone. Clinical toxicology (Philadelphia, PA) 2016;54:434-40.

26. Bao Y, Pan Y, Taylor A, et al. Prescription drug monitoring programs are associated with sustained reductions in opioid prescribing by physicians. Health Aff 2016;35:1045-51.

27. Hurstak EE, Kushel M, Chang J, et al. The risks of opioid treatment: Perspectives of primary care practitioners and patients from safety-net clinics. Subst Abuse 2017;38:213-21.

28. Jamison RN, Sheehan KA, Scanlan E, Matthews M, Ross EL. Beliefs and attitudes about opioid prescribing and chronic pain management: Survey of primary care providers. J Opioid Manag 2014;10:375-82.

29. Wiese HJC, Piercey RR, Clark CD. Changing prescribing behavior in the United States: Moving upstream in opioid prescription education. Clin Pharmacol Ther 2018;103:982-9.

30. Gwira Baumblatt JA, Wiedeman C, Dunn JR, Schaffner W, Paulozzi LJ, Jones TF. High-risk use by patients prescribed opioids for pain and its role in overdose deaths. JAMA Intern Med 2014;174:796-801.

31. United States Centers for Disease Control. CDC grand rounds: Prescription drug overdoses-A U.S. epidemic. MMWR Morb Mortal Wkly Rep 2012; 61:10-3.

32. Singhal A, Tien YY, Hsia RY. Racial-ethnic disparities in opioid prescriptions at emergency department visits for conditions commonly associated with prescription drug abuse. PLoS ONE 2016;11: e0159224.

33. Pletcher MJ, Kertesz SG, Kohn MA, Gonzales R. Trends in opioid prescribing by race/ethnicity for patients seeking care in US emergency departments. JAMA 2008;299:70-8.

34. Chen I, Kurz J, Pasanen M, et al. Racial differences in opioid use for chronic nonmalignant pain. J Gen Intern Med 2005;20:593-8.

35. Burgess DJ, Nelson DB, Gravely AA, et al. Racial differences in prescription of opioid analgesics for 
chronic noncancer pain in a national sample of veterans. J Pain 2014;15:447-55.

36. Cerdá M, Ransome Y, Keyes KM, et al. Prescription opioid mortality trends in New York City, 1990-2006: Examining the emergence of an epidemic. Drug Alcohol Depend 2013;132(1-2): 53-62.

37. Torres CA, Thorn BE, Kapoor S, DeMonte C. An examination of cultural values and pain management in foreign-born spanish-speaking hispanics seeking care at a federally qualified health center. Pain Med 2017;18: 2058-69.

38. Fishbain DA, Cole B, Lewis J, Rosomoff HL, Rosomoff RS. What percentage of chronic nonmalignant pain patients exposed to chronic opioid analgesic therapy develop abuse/addiction and/or aberrant drug-related behaviors? A structured evidence-based review. Pain Med 2008;9:444-59. 